\title{
The Role of Bilateral Prophylactic Mastectomy (BPMX) in Women at High Risk of Breast Cancer
}

\author{
A.D. Baildam
}

Women at high personal risk of breast cancer can be identified from family history using Claus data or from genetic testing for BRCAI and BRCA2 mutations. Women at such risk may be offered surveillance, entry into a chemoprevention trial such as IBIS, or bilateral prophylactic mastectomy (BPMX).

Recent published data on the use of Tamoxifen as a chemopreventative agent found reduction of risk in one study [1] but this was not confirmed in two other ongoing studies [2]. The theoretical risk reduction effect of BPMX receives some confirmation in a recent [3] study. Increasingly women are seeking surgery as a means to attempt to reduce their personal high risk.

The Manchester Breast Unit has an established strict protocol for the estimation of risk and selection of patients for surgery. All patients see a specialist geneticist, psychiatrist, oncologist and specialist breast surgeon. The aim of surgery is to reduce breast cancer mortality, relieve anxiety and allow women to live a normal life, which includes the aesthetic considerations of reconstructive surgery. There is however no standard operation and the extent of mastectomy in BPMX is variable between units.

Since 199543 women have undergone bilateral prophylactic mastectomy under the care of one surgeon. In 3 cases a conventional mastectomy was performed and in 2 with free nipple grafts. In 4 patients a total mastectomy was coupled with immediate reconstruction using a fixed volume submuscular permanent implant.
This did not give a reconstructive result of sufficiently high quality, and subsequently a technique was developed of total glandular mastectomy coupled with mastopexy and breast skin preservation. In 34 cases tissue expander devices were placed at the same time as mastectomy and inflated over 6 months to be replaced by permanent implants together with recreation of the inframammary fold as a second stage. In 2 cases immediate bilateral tram flaps were performed.

In 35 women bilateral nipple areola complexes (NAC) were preserved on de-epithelialised skin bridges, and in one case there was total NAC loss. In 4 women there was tissue expander device failure but in only one woman of 38 was an implant lost due to infection

The pathology found benign tissue only in 35 women; in 4 there was lobular carcinoma in situ and in 2 ductal carcinoma in situ. In a further 2 women ductal carcinoma in situ was associated with infiltrating duct carcinoma. All women with malignant disease had normal mammography preoperatively, but were diagnosed before operation by cytology and MRI scan.

BPMX is still an operation under development. If its place is to be established in the management of high-risk women then women should be treated according to selection and management by a strict protocol, consistent surgical technique and follow-up in specialist centres.

\section{References}

[1] Fisher, B., et al. Tamoxifen for prevention of breast cancer: report of the National Surgical 
Adjuvant Breast and Bowel Project P1 Study. J. Nat. Can. Inst. 80(18), (1998) 1371-1388.

[2] Pritchard, K.I. Tamoxifen effective in prevention of breast cancer. Lancet 352, (1998) 80-81.
[3] Hartmann, L.C., et al. Efficacy of bilateral mastectomy in women with a family history of breast cancer. New Eng. J. Med. 340, (1999) 7784. 


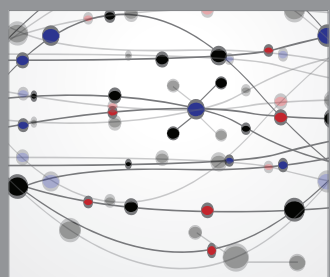

The Scientific World Journal
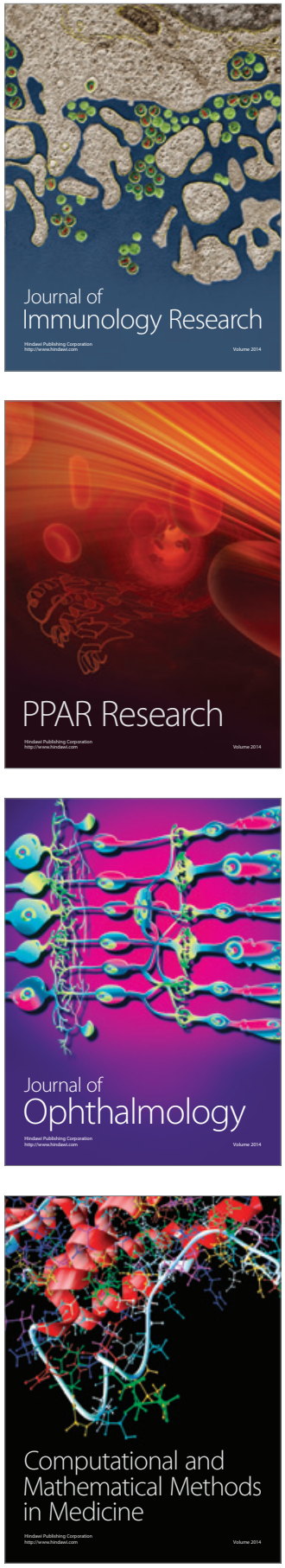

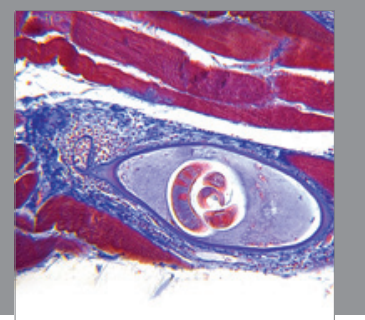

Gastroenterology

Research and Practice
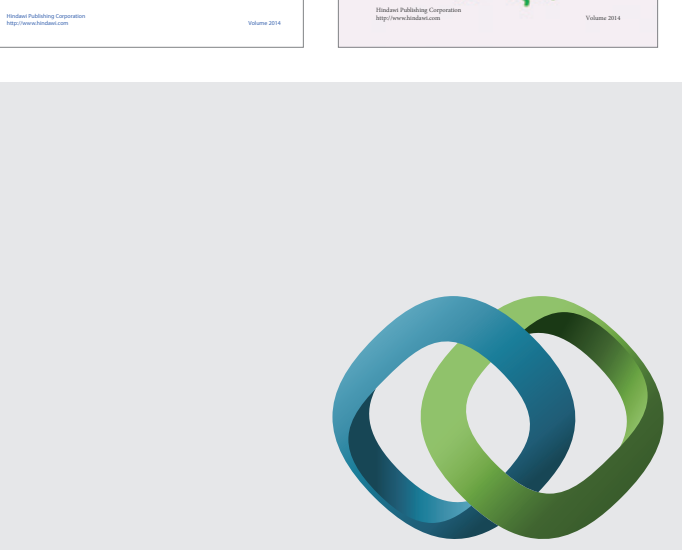

\section{Hindawi}

Submit your manuscripts at

http://www.hindawi.com
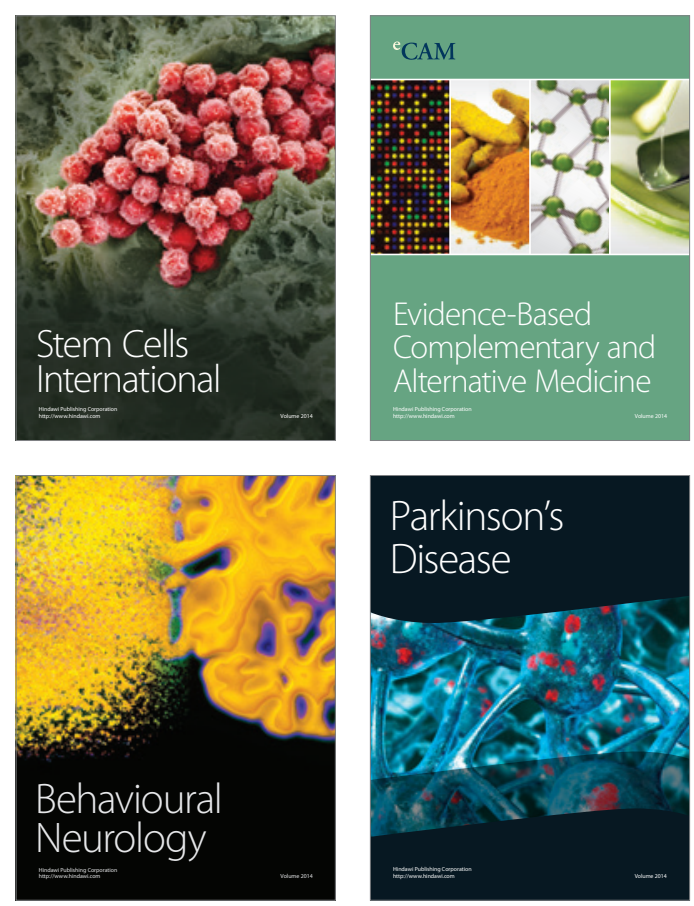

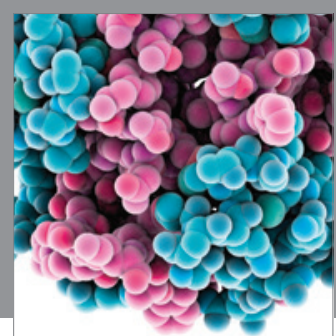

Journal of
Diabetes Research

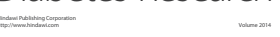

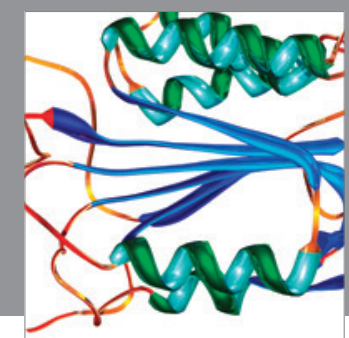

Disease Markers
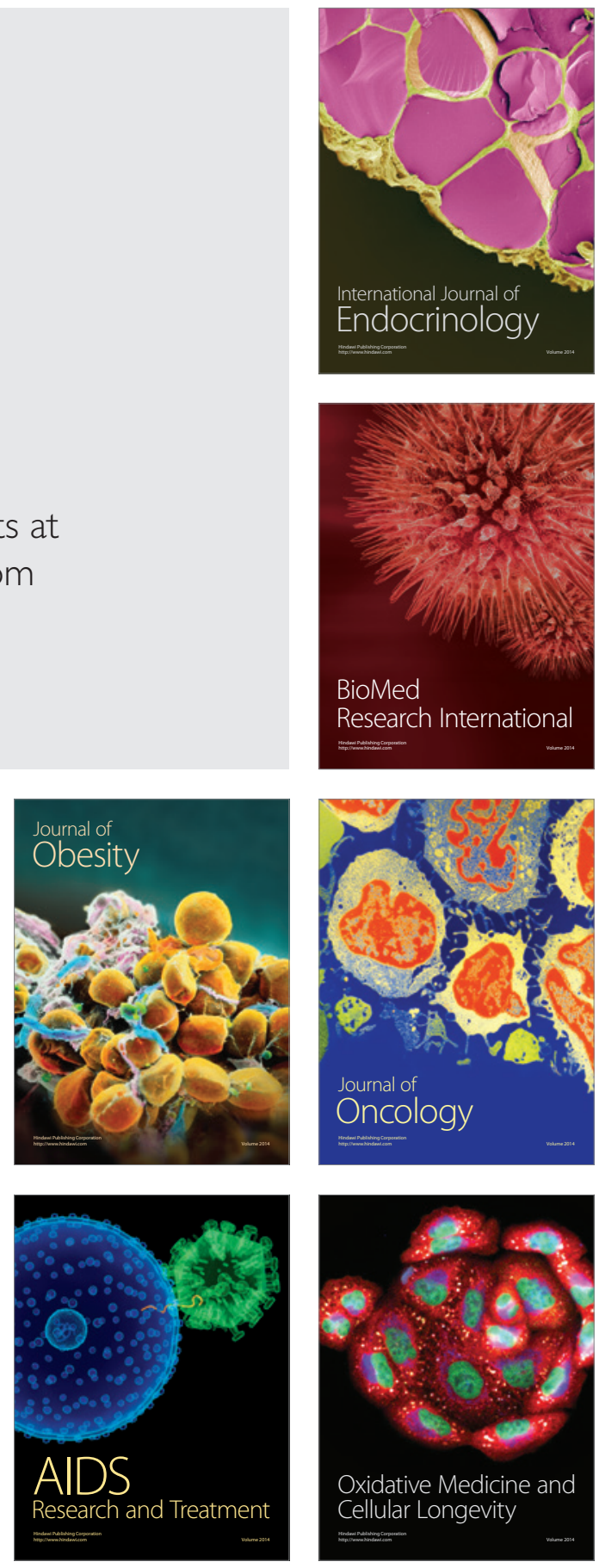